\title{
Recursive Definitions \\ in Type Theory
}

\author{
R.L. Constable \\ N.P. Mendler \\ TR 85-659 \\ January 1985
}

Department of Computer Science Cornel1 University

Ithaca, New York 14853 


\title{
RECURSIVE DEFINITIONS IN TYPE THEORY
}

\author{
R.L. Constable \\ N.P. Mendler \\ Computer Science Department \\ Cornell University \\ Ithaca, N.Y. 14853
}

Abstract

The type theories we consider are adequate for the foundations of mathematics and computer science. Recursive type definitions are important practical ways to organize data, and they express powerful axioms about the termination of procedures. In the theory examined here, the demands of practicality arising from our implemented system, Nuprl, suggest an approach to recursive types that significantly increases the proof theoretic power of the theory and leads to insights into computational semantics.

We offer a new account of recursive definitions for both types and partial functions. The computational requirements of the theory restrict recursive type definitions involving the total functionspace constructor $(\rightarrow)$ to those with only positive occurrences of the defined typed. But we show that arbitrary recursive definitions with respect to the partial function-space constructor are sensible. The partial function-space constructor allows us to express reflexive types of Scott's domain theory (as needed to model the lambda calculus) and thereby reconcile parts of domain theory with constructive type theory.

ACM subject classification: F.3.1 [Logics and Meaning of Programs]: Specifying and Verifying Reasoning about Programs. I.1.2 [Artificial Intelligence]: Automatic Programming, Deduction and Theorem Proving.

General Terms: Languages, Theory, Verification

Key Words and Phrases: Automated logic, domain theory, foundations of computing theory, inductive definitions, lambda calculus models, program verification, programming language design, program synthesis, partial recursive functions, recursive function theory, recursive data types, semantics of programming languages, type theory.

This work was supported in part by NSF grant MCS81-04018 


\section{Introduction}

\subsection{Context and Motivation}

To understand our reasons for studying recursive definitions in type theory, one should view that theory as an attempt to provide a comprehensive formal logical calculus in which to conduct rigorous programming. We have in mind a language rich enough to precisely specify programming problems and explain their solutions; so the calculus contains a usable programming language as an integral part. $^{\dagger}$ From our experience in designing and using logics of this variety, called programming logics [12], we have come to believe that type theory is very good for the task. One might find this immediately plausible by observing that the concept of type is to computing practice what the concept of set is to classical mathematical practice. That is, type is one of the central foundational concepts in programming, program is the other.

For us then, type theory is a comprehensive formal theory for expressing computational reasoning. The first type theory, that of Principia Mathematica, is such a theory for noncomputational mathematics. Specifically, we are interested in the type theory underlying the programming system Nuprl $[11,13,39]$ but these ideas would apply also to the theories of Martin-Löf [31], Girard [21] and Reynolds [41]. A different approach to these questions was taken in the theory V3 [15]. In all of these theories it is possible to define a sufficiently rich notion of recursive type and recursive function. Nevertheless, none of these theories provides an account which is as convenient and as transparent as the one offered here. It is sometimes for lack of convenience and clarity of central concepts that a theory fails to attract adherents.

\subsection{An Informal Account}

Nature of Type Definitions

Every object of concern to us is represented by symbols. Some of these symbols are canonical, such as $0,1,2$, and some are not, such as $0+1$. The canonical terms are those which are irreducible and denote specific objects. The noncanonical can be further reduced, and are said to denote the

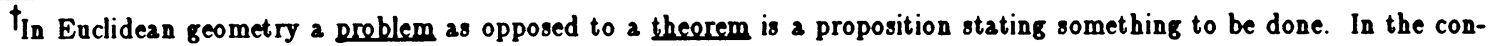
structive setting problems and theorems are nearly the same.
} 
canonical terms to which they reduce. When we specify a new object, we must provide a canonical name for it. Rarely is the name constructed in isolation, but rather we have in mind a method of constructing a pattern of canonical names, as with $0,0^{\prime}, 0^{\prime \prime}$, etc. Such methods denote types. Sometimes we agree that two canonical names will denote the same object, for example $2 / 4$ is the same rational number as $1 / 2$. Thus to completely specify a type, a definition of equality between canonical names is given. A theory of types is concerned with describing the methods of construction and the conditions for equality. This presentation of the meaning of type theory follows more or less Per Martin-Löf's [31] account of Errett Bishop [4]. These ideas have a long history in the work of L.E.J. Brouwer [6], Bertrand Russell [42], Alonzo Church [9], and W. Quine [40], among others.

The type theories we consider have constructors for defining the disjoint union of $A$ and $B$, denoted $\mathrm{A} \mid \mathrm{B}$, the cartesian product, denoted $\mathrm{A} \times \mathrm{B}$, and the type of all (total computable) functions from $A$ to $B$, denoted $A \rightarrow B$.

Recursive Definitions

Recursive and inductive methods are ways of specifying types. For example, we interpret the definition:

(*) define type $\mathrm{T}$ by $\mathrm{A} \mid(\mathrm{T} \times \mathrm{A})$

as a method which says:

(**) To construct an element of type $T$, construct either an element of $A$ or construct an element of $T$ and an element of $A$.

Such definitions are natural when we conceive of the process of construction top-down, that is in a goal oriented manner.

If pairs in $A \times B$ are denoted $a, b$ for $a$ in $A, b$ in $B$; and if inl and inr are the "into the left disjunct" and "into the right disjunct" mappings respectively so that inl(a) and $\operatorname{inr}(\mathrm{b})$ are in $\mathrm{A} \mid \mathrm{B}$; then the following are elements of the recursive type in $(*)$ :

inl(a)

inr(inl(a),a)

$\operatorname{inr}(\operatorname{inr}(\operatorname{inl}(\mathbf{a}), a), a)$ 
Such a type has a natural inductive structure. The base case elements are of the form inr(a) for a in $A$, and the inductive case element, have the form inl(t,a) for $t$ in $T$ and $a$ in $A$.

\section{Notation}

The notation for a recursive type should suggest the rule that elements of the defined type, say $T$, are built using the pattern of the defining expression. We might write $(T:=A \mid T \times A)$ or use the Algol 68 style, type $\mathrm{T}=\mathrm{A} \mid \mathrm{T} \times \mathrm{A}$. Whatever the notation, it is complicated by the possibility that the defined type $\mathrm{T}$ may depend on parameters. For example, $\mathrm{A}$ might be a regarded as a parameter; to emphasize this, let us use a name that connotes variables, say

define $\mathrm{T}(\mathbf{y})$ by $\mathbf{y} \mid \mathrm{T}(\mathbf{y}) \times \mathbf{y}$.

We might also write $\lambda y . \mu T .(y \mid T(y) \times y)$ following $[30]$ or type $t(y)=y \mid T(y) \times y . T h e s e$ parameterized definitions do not specify a type until a value is given for the parameter. The parameter may enter in other ways, for example, suppose there is a type of the form [1,n] which denotes the integers 1 up to $n$. Consider:

define $T(\mathbf{n})$ by $[1, \mathbf{n}] \mid \mathrm{T}(\mathbf{n}+\mathbf{1})$.

We have adopted the notation that $\operatorname{rec}(t, x . T)(a)$

defines a recursive type parameterized by $x$ and specialized to $a$. The expression $T$ denotes a type which can use $t$ and $x$ in its formation. We say that $t$ and $x$ are bound in $T$. Complete binary trees with leaves of type integer are defined by $\operatorname{rec}(t, x . x \mid t(x) \times t(x))($ int $)$. This is equivalent to rec(t. int | $t \times t)$, which informally we would have written define $t$ by int $\mid t \times t$. A typical element of the type is $\operatorname{inr}(\operatorname{inr}(\operatorname{inl}(0), \operatorname{inl}(1)), \operatorname{inl}(1))$.

We prefer this notation to the more common $\lambda y . \mu t . T$ or to typet(y)=T for three reasons. The two are stylistic, we want to treat rec and fix alike and we think of fix as an operator or functionals (like FIX in LCF [22]), so we would be inclined to write $\mu$ t.( $(\lambda y . T)$ as opposed to $\lambda y . \mu t . T$ if we were to adopt the mu operator notation. Furthermore, in the case of mutual recursion, $\operatorname{say} \operatorname{rec}(t, x$. $T ; s, y$. $U)$ the variables $c, y$ are bound only in $T$ and $U$ respectively whereas $t$ and 8 are bound in both $T$ and 
$U$, so $x$ and $y$ have smaller scope and are written closer to their scopes. The third reason is more technical; we do not in fact treat $\operatorname{rec}(t, x$. $T)$ as a function from some type $A$ into types because the principal use of the notation arises in denoting types, as in $\operatorname{rec}(t, x . T)(a)$, and treating this as a function application would cause extra steps of reasoning in most uses. Since rec(t,x. $T)(a)$ is not a function application, we might be better advised to write $\operatorname{rec}(t, x . T ; a)$. Presently we think that this overloads the work done inside the parentheses in the case of mutual recursion and complicates notation for substitution womewhat, but it is an attractive possibility.

The notation for specifying one of $n$ mutually recursive types is $\operatorname{rec}\left(t_{1}, x_{1} . T_{1} ; \ldots ; t_{n}, x_{n} . T_{n} ; t_{i}\right)\left(a_{i}\right)$ for $i=1, \ldots, n$. The $t_{1}$ have the entire expression as scope and are bound anywhere in the scope, but the $x_{1}$ has only $T_{i}$ as scope and is bound only in $T_{i}$ Thus in $\operatorname{rec}\left(t_{1}, x_{1}\right.$. int $\left.\mid t_{1}\left(x_{1}\right) ; t_{2}, x_{2} . t_{1}\left(f\left(x_{1}, x_{2}\right)\right) \times t_{2}\left(x_{2}\right) ; t_{2}\right)(a)$ the occurrence of $x_{1}$ in $f\left(x_{1}, x_{2}\right)$ is free, all other occurrences of $x_{1}$ and $x_{2}$ are bound.

The approach to recursive types suggested above is by now quite standard, its ancestors can be found at least as far back as Hoare [24] and a detailed rigorous account is given in ML [22] and in Cartwright [7] to name two basic sources. This kind of type definition can be built upon the reference mechanism of Algol 68 or upon the pointer mechanism of Pascal, ADA, etc. The presentation here is simply a manifestation of these basic ideas in the setting of a constructive type theory.

\section{Recursive Definition of Function Spaces}

The concept of a recursive type involving the function space constructor is far more delicate than the above case and is not treated in Hoare or Cartwright for example, although Algol 68 does provide a mechanism to represent such types as

$$
\text { mode } t=\operatorname{proc}(t) t
$$

where proc(A)B is the mode of all (partial) functions from mode A to mode B (see McGettrich [34]), and $M L[22]$ provides such types, written absrectype $t=t \rightarrow t$.

There have been numerous approaches to recursive type definitions involving the function space. The best known are probably Scott's use of lattices and domain theory because they provided the first lambda calculus models [44]. This approach is intimately connected with partial functions 
and will be discussed later. Another approach based on partial functions is MacQueen and Sethi [30] where types are treated as ideals. Function spaces also arise in accounts of generalized inductive definitions, both classically as in Feferman [20] and constructively as in Kriesel [27] and Martin-Löf [32]. These accounts are based on total functions and are close to the one given here, so it might be more suggestive to call these inductive types. But we will see that the computer science terminology is justified once we introduce the partial function type $\mathrm{A} \sim>\mathrm{B}$.

When one first approaches recursive types defined over the function spaces, such as rec $(t . t \rightarrow t)$ or $r e c(t . t \rightarrow A)$, it appears that these notations do provide a prescription for building objects and that the lambda expressions provide the required canonical objects. In fact, naively thinking of $\operatorname{rec}(t, x . T)$ as a prescription for building objects appears to make sense regardless of the form of $\mathrm{T}$ as long as it is defined under the assumption that $t$ is a type and $x$ belongs to some type. But let us examine the simple special case $\operatorname{rec}(t . t \rightarrow A)$ where $A$ is any type.

Let $T=\operatorname{rec}(t . t \rightarrow A)$, then to build an object of type $T$, we must build one of type $T \rightarrow A$. This is a function type, so we assume $x: T$ and build an element of $A$. This assumption amounts to $x: T \rightarrow A$. To use $x$ we must build an element of $T$. But $x$ itself is such an element, thus $\lambda x \cdot x(x)$ belongs to $\mathrm{T}$. Now then, $(\lambda \mathbf{x} \cdot \mathbf{x}(\mathbf{x}))(\lambda \mathbf{x} \cdot \mathbf{x}(\mathbf{x}))$ belongs to $\mathrm{A}$.

If $\mathrm{A}$ is void, then the above derivation shows an inconsistency. But even if $\mathrm{A}$ is inhabited, the element $(\lambda x \cdot x(x))(\lambda x \cdot x(x))$ yields a nonterminating computation. So $\lambda x \cdot x(x)$ is not a total function as is required to be an element of a function space such as $\operatorname{rec}(t . t \rightarrow t) \rightarrow A$.

\section{Analysis of the Function Case}

Why does the example rec $(t . t \rightarrow A)$ cause problems? One answer is that the rule for function introduction and the rule for recursive type introduction combine in such a way as to produce a vicious circle. To see this, consider the function rule in this top down form:

To build an element of $A \rightarrow B$, assume $x$ is in $A$ and build an element $b$ of $B$. The element of $A \rightarrow B$ is then $\lambda x . b$. 
Now the rule for $\operatorname{rec}(t . t \rightarrow A)$ says, assume $x$ is in $\operatorname{rec}(t . t \rightarrow A)$ and build a term $b$ in $\operatorname{rec}(t$. $t \rightarrow A)$. But by assuming $x: \operatorname{rec}(t . t \rightarrow A)$ we are already assuming that we know $\operatorname{rec}(t . t \rightarrow A)$, the type we are trying to build. This is similar to the situation underlying Russell's paradox, and indeed the term $\{x \mid \neg x(x)\}$ is similar to $\lambda x . x(x)$. We see here a genuine and instructive parallel because the rule for recursive types is a rule to determine the existence of functions which is an issue of the same force as determining the existence of sets.

Mendler has shown that whenever $t$ is not positive in $T$, then rec $(t . T)$ contains terms whose computations fail to terminate. For example $t$ is negative in $t \rightarrow t$, in $t \rightarrow A$, in $A \rightarrow(t \rightarrow B)$ and in general whenever $t$ is in an odd number of antecedents. This suggests that rec(t. $T)$ might be sensible only when $\mathbf{t}$ does not occur negatively in $\mathrm{T}$. So far we have only been able to show that whenever $t$ does not occur as an antecedent to an implication, then the definition is reasonable, at least in the sense that the function elements of the recursive type are total. So in terms such as $A \rightarrow t$, $A \rightarrow(B \rightarrow t)$, etc. $t$ occurs in a safe way. In section III we say that $t$ occurs positively in $T$ under these conditions, but not all nonnegative occurrences, such as $(t \rightarrow A) \rightarrow B$ are positive.

If one begins with the concept of a partial function, as in $[29,30,43]$, recursive types are analyzed in a very different way. The element $(\lambda x \cdot x(x))(\lambda x \cdot x(x))$ is assigned a value, say $\perp$, and the analysis above shows that $\perp$ must occur in every type. Such a conclusion is at variance with the propositions-as-types principle used in organizing AUTOMATH [18], Martin-Löf type theories [31] and Nuprl $[11,13,39]$. Moreover, in constructive logic the concept of a total computable function is more basic than that of a partial function and is indeed needed to define it, as will be suggested in this paper. So it is interesting to first clarify the concept of recursive definitions involving the total function spaces.

\section{Significance of Partial Functions}

Partial functions play an important role in computing theory for a number of reasons. We have just seen above that they figure in an analysis of the difficulties with $\operatorname{rec}(t . t \rightarrow A)$. They are also the main organizing concept in Basic Recursive Function Theory [25], e.g. partial functions are taken as the mathematical meaning of algorithm. They are also natural in most programming 
languages, e.g. a Lisp interpreter in inherently partial. Some of the interesting heuristics written in ML to find Nuprl proofs are partial. In fact, the correctness of programs is often stated in two parts; first partial correctness and then termination. A program is partially correct if it satisfies its specifications under the assumption that it halts.

Partial functions are central in Scott's theory of domains [43-47], and the principal rule of inference in LCF [22], a logic based on domains, is the fixed point induction rule for (admissible) assertions about partial functions. Even though type theories and set theories can adequately define a concept of partial function, even in the case of constructive theories $[12,16]$, such definitions do not treat the functions directly as they are given in programming. For example, extra termination parameters are added, but domain theory does treat them directly.

We would like to have a convenient and natural account of partial functions in type theory in as far as they facilitate programming in it and in order to explain ordinary programming. We intend that our type theories provide not only a logically adequate foundation for computing but a practically adequate foundation as well in the sense that the formal theory can be used as a real programming language. This requires that the execution facilities be efficient and that the means of expression be natural.

One way to achieve such a theory in the context of type theory might be to import the domain concept. A number of people have been examining this question lately, including Per Martin-Löf, Gordon Plotkin, Samson Abramsky, Peter Aczel and others. One immediate observation already made above is that replacing types by domains spoils the propositions-as-types principle because the least element, $\perp$, inhabits every domain; thus truth of a proposition cannot correspond to inhabitation of type. Moreover, the continuity and monotonicity properties which are axiomatic for domains are typically derived notions in type theory. Lately Gordon Plotkin [38] has proposed a theory of domains without a least element. It will be interesting to see how such an approach relates to type theory. 


\section{Partial Functions in Type Theory}

We think that there is a direct way to treat partial functions in type theory. An example of this method appears in [12]. The idea is that with every partial function $f$ on $A$ into $B$ we associate a domain condition, $\operatorname{dom}(f)(x)$. The partial function then has the type $y:\{x: A \mid \operatorname{dom}(f)(x)\} \rightarrow B^{\dagger}$ The dom $(f)(x)$ condition is a recursive type of the theory. Here is an example of dom( $f)(x)$ for a simple recursive function written in a neutral notation:

$$
\begin{aligned}
& \mathrm{f}(\mathrm{x}):=\text { if } \mathrm{b}(\mathbf{x}) \text { then } \mathrm{g}(\mathbf{x}) \text { else } \mathbf{h}(\mathbf{f}(\mathrm{e}(\mathbf{x}))) f i \\
& \operatorname{dom}(f)(x)=\operatorname{rec}(d, x \cdot \operatorname{dom}(b)(x) \& \\
& (\mathrm{b}(\mathrm{x})=\text { true } \Rightarrow \operatorname{dom}(\mathrm{g})(\mathrm{x})) \\
& \&(b(x)=\text { false } \Rightarrow \operatorname{dom}(e)(x) \& d(e(x)) \& \operatorname{dom}(h)(f(e(x)))))(x)
\end{aligned}
$$

(The actual definition given in III.2 uses a conditional-and in place of $\Rightarrow$.)

In this setting we think of an algorithm as simultaneously specifying a domain and a function. But the algorithm is present directly and can perhaps be executed for elements about which we have no information that $\operatorname{dom}(f)(a)$. In these cases we cannot guarantee the properties of the algorithm.

We want our notation for algorithms to be natural, at least as natural as function definitions in algol-like or lisp-like programming languages. To this end we adopt a style similar to ML[22]. A partial recursive function, or algorithm, is written as fix $(f, x . F)$ where $F$ is an expression. The computation rule for fix $(f, x . F)$ is typeless, namely for any expression a fix $(f, x . F)[a]=F(a / x, f i x(f, x$. F)/f). It is important when we are discussing this concept in the setting of Martin-Löf's semantics to know that fix $(f, x . F)$ is a canonical form and evaluation is outermost.

\section{Concept of a Partial Function Type}

We want the partial functions from $A$ to $B$ to form a type, but we cannot define such a type with the existing constructors. So we introduce a new type $A \sim>B$ which could be used to define $A \rightarrow B$ by the condition $A \rightarrow B=\{f: A \sim>B \mid \forall x: A . \operatorname{dom}(f)(x)\} .^{\ddagger}$ Once this new type is available, we can use it in recursive definitions to give a coherent account of $\operatorname{rec}(t . t \sim>t)$. This sort of type allows us to treat reflexive types in the sense of Scott [43].

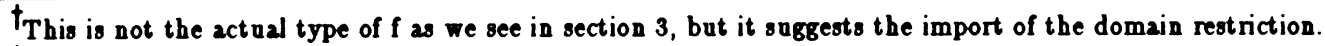

$f_{W e}$ do not in fact nse this definition in Nuprl because the total function space constructor, $\rightarrow$, is needed to define the meaning of logical operations such as $\forall x: A$. dom(f) (x).
} 


\section{Recursive Types}

\section{Common Patterns}

Inductive definitions of types and sets abound in computer science. For instance, the data type of trees which we used before is defined in Knuth [28] p. 305 as

"Let us define a tree formally as a finite set $T$ of one or more nodes such that

a) There is one specially designated node called the root of the tree, root(T); and

b) The remaining nodes (excluding the root) are partitioned into $m \geq 0$ disjoint sets $T_{1}, \ldots, T_{m}$ each of these sets in turn is a tree."

In logic, the set of formulas is usually defined inductively, for example the propositional formulas $F$ might be defined as:

(i) true, false are in $F$ called constants

(ii) variables $x_{1}, x_{2}, \ldots$ are in $F$

(iii) if $f, g$ are in $F$, then so are ( $f \& g)$ and ( $f V g)$.

(iv) nothing else is in F.

In algebra, we see inductive definitions of substructures generated by a subset of elements; for instance the subgroup of $G$ generated by elements $b_{1}, \ldots, b_{n}$ is the least subgroup $H$ of $G$ such that

(i) $b_{1}, \ldots, b_{n}$ are in $H$ and

(ii) if $x, y$ are in $H, x \cdot y^{-1}$ is in $H$ also.

In set theory and recursion theory, the concept of an ordinal number is typically defined inductively as in this definition of the order relation, $<_{0}$, on the constructive ordinal notations as the least binary relation $\mathrm{R}$ such that:

(i) $1 \mathrm{R} \mathrm{z}$

(ii) $x R y \Rightarrow x R 2^{y}$

(iii) $x \mathrm{R}$ y \& y R z $\Rightarrow x \mathrm{Rz}$

(iv) $\forall \mathrm{n}, \mathrm{m} .\left(\mathrm{m}<\mathrm{n} \Rightarrow \phi_{\mathrm{s}}(\mathrm{m}) \mathrm{R} \phi_{\mathrm{s}}(\mathrm{n})\right) \Rightarrow \forall \mathrm{n} .\left(\phi_{\mathrm{s}}(\mathrm{n}) \mathrm{R} 5^{\mathrm{x}}\right)$

The ordinal notations, 0 , are $\{x \mid \exists y \quad x<a y\}$.

Post production systems are a formalism for writing a certain kind of inductive definition; they arose as a generalization of the definition pattern of the classes of formulas and proofs used in Principia Mathematica. Similarly the concept of a context free or context sensitive language generated by a grammar $G$ is an instance of an inductive definition. That is, the language, $L(G)$ is defined in terms of the transitive closure of the derivability relation $\rightarrow$. Generally the transitive closure, $R^{*}$, of a relation $R$ is defined to be the least relation $S$ such that 


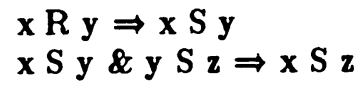

All of these kinds of inductive definition will be rendered in our theory as instances of recursive types. We now proceed to describe the syntax and proof rules for these types and give examples of each kind.

\section{Syntax}

\section{The Recursive Form}

We introduce a new syntactic form to denote mutually recursive types. The simplest special case is $\operatorname{rec}(t . T)$ as in $\operatorname{rec}(t$. int $\mid(t \# t))$. For parameterized types we use rec(t,x. $T)(a)$ as in $\operatorname{rec}(t, x$. $x \mid(t \# t))($ int $)$. In full generality we use the notation $\operatorname{rec}\left(t_{1}, x_{1} . T_{1} ; \ldots ; t_{n}, x_{n} . T_{n} ; t_{1}\right)(a)$ for $i$ in $\{1, \ldots, n\}$. This represents the mutually recursive definition of $t_{1}\left(x_{1}\right), \ldots, t_{n}\left(x_{n}\right)$. Any of the $x_{1}$ parameters can be omitted. The $t_{1}$ are bound in $T_{1}, \ldots, T_{n}$ but $x_{1}$ is bound only in $T_{1}$. Here is a scheme for three mutu-

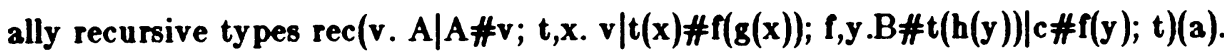

\section{Nonrecursive Forms}

In order to precisely define the syntax of recursive types, we must specify the underlying type expressions. We use Nuprl for this $[13,39]$ but the account given here is reasonably self contained. The base types are int and void. The type constructors are $\mid, \#, \rightarrow$, list, // and \{\} , but the last three constructors, for lists, quotients and sets do not introduce a new complexity into the definition, so only the first three are used here. ${ }^{\dagger}$ The rules for defining these types are presented in the Nuprl style in terms of sequents.

$$
\mathrm{H} \gg \mathrm{T} \text { in } \mathrm{U}_{1}
$$

where $H$ is a list of typings, of the form $x_{1}: A_{1}, x_{2}: A_{2}\left(x_{1}\right), \ldots, x_{n}: A_{n}\left(x_{1}, \ldots, x_{n-1}\right), T$ is a type and $U_{1}$ is a universe. For our purposes a universe can be considered as a type whose elements are types, and we will only use one universe, $U_{1}$. This sequent means that $T$ is a type in the $U_{i}$ under the assumptions in $H$. Here we are using the notation $A\left(x_{1}, \ldots, x_{n}\right)$ to indicate that the free variables $x_{1}, \ldots, x_{n}$ may occur

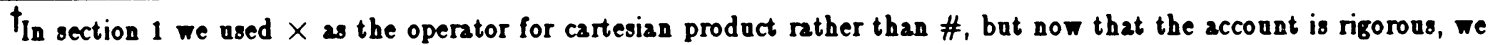
want to use the notation from the implemented Nuprl theory [30].
} 
among the free variables of $A$. We will write $A(t / x)$ to denote the substitution of $t$ for all free occurrences of $x$. A formation rule is presented in this form

$\mathrm{H}>\mathrm{P} \mid \mathrm{B}$ in $\mathrm{U}_{1}$

1. $\mathrm{H}>>\mathrm{A}$ in $\mathrm{U}_{1}$

2. $\mathrm{H}>>\mathrm{B}$ in $\mathrm{U}_{1}$

This rule is read, $A \mid B$ is a type in $U_{1}$ under assumptions $H$, if $A$ is in $U_{J}$ under assumptions $H$, and $B$ is in $U_{k}$ under assumptions $H$. Here are the rules for type formation.

1. disjoint union

$\mathrm{H} \gg \mathrm{A} / \mathrm{B}$ in $\mathrm{U}_{1}$ by intro

1. $H>P$ in $U_{1}$

2. $\mathrm{H}>>\mathrm{B}$ in $\mathrm{U}_{1}$

2. dependent product

$H>>x: A \# B(x)$ in $U_{1}$ by intro

1. $H>>A$ in $U_{1}$

2. $\mathrm{H}, \mathrm{x}: \mathrm{A}>>\mathrm{B}(\mathrm{x})$ in $\mathrm{U}_{1}$

3. function space

$H>>x: A \rightarrow B(x)$ in $U_{1}$ by intro

1. $\mathrm{H}>>\mathrm{A}$ in $\mathrm{U}_{1}$

2. $\mathrm{H}, \mathrm{x}: \mathrm{A}>\mathrm{A}(\mathrm{x})$ in $\mathrm{U}_{1}$

In the expressions $x: A \# B(x)$ and $x: A \rightarrow B(x)$, occurrences of $x$ in $B$ are bound.

With the disjoint union, the dependent product, and with integers there are compound expressions used in the introduction and elimination rules. They are $\operatorname{inl}(a), \operatorname{inr}(b), \operatorname{decide}\left(d ; u . t_{1} ; v \cdot t_{2}\right)$, $\langle a, b\rangle$, spread(p;u,v.t) and ind(e;b;u,v.h). These forms satisfy the following computation rules.

1. decide(inl(a);u. $\left.t_{1} ; v \cdot t_{2}\right)=t_{1}(a / u)$

decide(inr(b);u.t $\left.t_{1} ; v . t_{2}\right)=t_{2}(b / v)$

2. $\quad \operatorname{spread}(<a, b>; u, v . t)=t(a / u, b / v)$

3. $\quad$ ind $(0 ; b ; \mathbf{u}, \mathbf{v} \cdot \mathbf{h})=\mathbf{b}$

$\operatorname{ind}(x+1 ; b ; u, v . h)=h(x / u, i n d(x ; b ; u, v . h) / h)$

The expressions inl(e), inr(e) are used to inject $e$ into the left or right sides respectively of a disjoint union, and $\langle\mathrm{a}, \mathrm{b}\rangle$ is used to build a pair in a dependent product. 


\section{Restrictions On Recursive Forms}

In forming rec(t. $T)$ and other recursive types, it is necessary to ensure that $t$ occurs only positively in $\mathrm{T}$, for reasons discussed in the introduction. The basic restriction is that $\mathrm{t}$ not occur as the domain type of a function space, as in $t \rightarrow i n t$. We can prevent this by saying $t$ is positive in $T$ provided: if $T$ is $T_{1} \mid T_{2}$, then $t$ is positive in $T_{1}$ and in $T_{2}$ if $T$ is $x: T_{1} \# T_{2}$, then $t$ is positive in $T_{1}$ and $T_{2}$; if $T$ is $x: T_{1} \rightarrow T_{2}$ then $t$ does not occur in $T_{1}$ and is positive in $T_{2}$. But this definition is complicated by the mutually recursive form and by the presence of the elimination forms, decide, spread and ind. Here is the general definition.

Definition: The recursive definition form $\operatorname{rec}\left(t_{1}, x_{1}, T_{1} ; \ldots ; t_{n}, x_{n} \cdot T_{n} ; t_{1}\right)(a)$ is positive in $t_{1}, \ldots, t_{n}$ provided the $t_{1}$ occur only positively in the $T_{j}$. Say that $t$ occurs positively in $T$ (or say for short $t$ is positive in $\mathrm{T}$ ) under these conditions. If

1. T is int or void or a type variable,

2. $T$ is $A \mid B$, and $t$ is positive in $A$ and in $B$,

3. $T$ is $x: A \# B(x)$, and $t$ is positive in $A$ and in $B(x)$,

4. $T$ is $x: A \rightarrow B(x)$, and $t$ does not occur in $A$ and is positive in $B(x)$,

5. T is $\operatorname{rec}\left(s_{1}, y_{1}, S_{1} ; \ldots ; s_{m}, y_{m} \cdot S_{m} ; s_{j}\right)(b)$ and $t$ is positive in $S_{k}$ for $k=1, \ldots, m$.

6. T is decide(d;u. $\left.e_{1}, v . e_{2}\right)$, and $t$ is positive in $d, e_{1}, e_{2}$ and if $t$ occurs in $d$, then $u, v$ are positive in $e_{1}, e_{2}$ respectively,

7. $T$ is $\operatorname{spread}(p ; u, v . q)$, and $t$ is positive in $p$ and $q$ and if $t$ occurs in $p$, then $u, v$ are positive in $q$,

8. T is ind(e;b;u,v.h), and $t$ is positive in $e, b$ and $h$, and if $t$ occurs in $b$, then $v$ is positive in $h$,

9. $T$ is $f(a)$, and if $f$ is a variable, then $t$ does not occur in $a$; otherwise if $f$ is $\lambda x$ x.b, then $t$ is positive in $a$ and $b$, and $x$ is positive in $b$. If $f$ is noncanonical, then $t$ is positive $f$ and $a$.

We also need to say when $t$ occurs positively in introduction forms involving types such as inl $(t \rightarrow A)$ or $<t, A>$. (In M-L [31] one would also have to consider such forms as $(t \rightarrow A)^{\prime}$ where ' is the successor operation.) We say that $t$ is positive in $T^{\prime}, \operatorname{inl}(T), \operatorname{inr}(T),\left\langle T_{1}, T_{2}\right\rangle$ provided it is positive in $T$. 


\section{Proof Rules}

General

The proof rules for recursive types are organized, as nearly all rules are, into the categories of formation, introduction, and elimination. But the category of elimination is subdivided into ordinary elimination and induction. In every rule it must be the case that the recursive type expression is positive. This is checked during parsing. Optional parts of a rule are enclosed in \|. We assume that all hypotheses are numbered but we do not display the number.

\section{Formation Rules}

1. $H>\operatorname{rec}\left(t_{1}, x_{1}, T_{1} ; \ldots ; t_{n}, x_{n} . T_{n} ; t_{1}\right)(a)$ in $U_{k}$

by intro using $A_{1}, \ldots, A_{n}$ at $U_{k}\left[n e w ~ x, y_{1}, \ldots, y_{n}\right]$

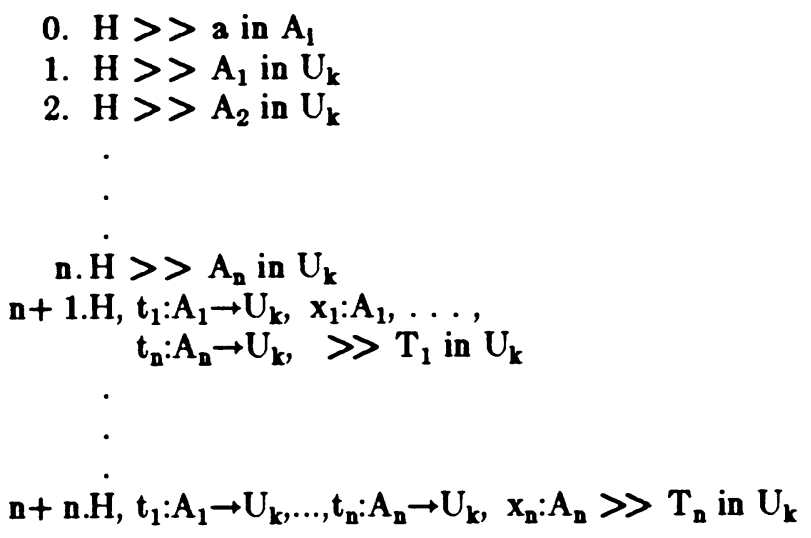

The new $y_{1}$ are used to rename $x_{1}$ if $H$ already contains a declaration of $x_{1}$.

The hypotheses $t_{1}: A_{1} \rightarrow U_{k}$, need not be named because they are used only in eliminations in which case their number is a sufficient reference. When the $x_{1}$ do not appear, then "using $A_{1}$ " need not occur either. A very simple special case of this rule is:

$H>>\operatorname{rec}(t . T)$ in $U_{1}$ by intro at $U_{1}$

$\mathrm{H}, \mathrm{t}: \mathrm{U}_{1}>\mathrm{T}$ in $\mathrm{U}_{1}$

Here are simple examples of these rules:

ex1-F: $>>\operatorname{rec}(t . t)$ in $U_{1}$ by intro at $U_{1}$ $t: U_{1}>>t$ in $U_{1}$ 
ex2-F: $>>\operatorname{rec}(t, x . t(x))(5)$ in $U_{1}$ by intro using int at $U_{1}$ new $z$

$>>5$ in int

$t:$ int $\rightarrow U_{1}, x:$ int $>>t(x)$ in $U_{1}$

ex3-F: $>>\operatorname{rec}(t, x . x \mid x \# t(x))($ int $)$ in $U_{1}$ by intro using $U_{1}$ at $U_{1}$ new $z$ $>>$ int in $U_{1}$ $t: U_{1} \rightarrow U_{1}, x: U_{1}>>x \mid x \# t(x)$ in $U_{1}$

We can think of $\operatorname{rec}(t, x . x \mid x \# t(x))(A)$ as the type list(A).

ex4-F: $>>\operatorname{rec}(t . A|t \# f ; f . B| f \# t ; t)$ in $U_{1}$ by intro at $U_{1}$

$t: U_{1}, f: U_{1} \gg A \mid t \# f$ in $U_{1}$

$t: U_{1}, f: U_{1}>B \mid f \# t$ in $U_{1}$

Introduction Rules

2. $H>>\operatorname{rec}\left(t_{1}, x_{1} \cdot T_{1} ; \ldots ; t_{n}, x_{n} \cdot T_{n} ; t_{i}\right)(a)$ by intro

$H>T_{i}\left(\operatorname{rec}\left(-; t_{j}\right) / t_{j}, a / x_{i}\right)$

The expression rec(-; $\left.t_{j}\right)$ abbreviates $\operatorname{rec}\left(t_{1}, x_{1} . T_{1} ; \ldots ; t_{n}, x_{n} . T_{n} ; t_{j}\right)$ throughout.

Here are examples of the application of this rule:

ex1-I: $>>\operatorname{rec}($ t. t $)$ by intro

$>\operatorname{rec}(t . t)$

One can see from one application that it will be impossible to build an element of either rec(t.t) or $\operatorname{rec}(\mathbf{t}, \mathbf{x . t}(\mathbf{x}))(5)$.

ex2-I: $>>\operatorname{rec}(t, x . t(x))(5)$ by intro

$>>\operatorname{rec}(t, x \cdot t(x))(5)$

ex3-I: $>>\operatorname{rec}(t, x \cdot x \mid x \# t(x))($ int $)$ by intro

$>>$ int|int\#rec(t,x. $x \mid x \# t(x))($ int $)$

\section{Elimination Rules}

3. $H, y: \operatorname{rec}\left(t_{1}, x_{1} \cdot T_{1} ; \ldots ; t_{n}, x_{n} \cdot T_{n} ; t_{i}\right)(a), H^{\prime}>>T$ by elim $y$

$H, y: T_{i}\left(\operatorname{rec}\left(-; t_{j}\right) / t_{j}, a / x_{i}\right), H^{\prime}>T$

Here are examples of the simple elimination rule: 
ex1-E: $y: \operatorname{rec}(t . t)>>$ int by elim $y$

$$
y: \operatorname{rec}(t . t)>>\text { int }
$$

So the elimination rule makes no progress in this case.

ex3-E: $y: \operatorname{rec}(t, x . x \mid x \# t(x))>>$ int by elim $y$ $y: \operatorname{int} \mid$ int \#rec(t,x. $x \mid x \# t(x))($ int $)>>$ int

One might try to build an integer for the goal by another elimination on $y$. In the subcase $y$ :int $>>$ int the method succeeds, but in the case $y:$ int\# $\operatorname{rec}(-)>>$ int it would not be possible to continue using elimination on the recursive type indefinitely; eventually another rule such as explicit introduction would be necessary.

\section{Induction}

The most general form of elimination on a recursive type is an induction rule. The induction is on the process of building terms and makes sense because a term can be constructed in rec(-) only by a finite number of applications of the introduction rules. Here is the rule for the simple case of a parameter free recursive type.

$$
\begin{gathered}
H, x: r e c(t . T)>>G \text { by ind on } x \text { at } U_{1} \text { new } X: U_{1}, y, h \\
\text { H, X: } U_{1}, h:(x: X \rightarrow G), x: T(X / t)>>G
\end{gathered}
$$

The new type $\mathrm{X}$ represents a completely unknown type of the right level which is used to indicate an appeal to the induction hypothesis in the decomposition of $T(X / t)$. The induction hypothesis is $h:(x: X \rightarrow G)$.

Notice that while we can prove nothing by simple elimination on rec(t. $t)$, we can prove anything by induction on that type. For instance

$$
\begin{gathered}
\text { x:rec(t. t) }>>\text { void by ind } x \text { at } U_{1} \text { new } X, h, y \\
X: U_{1}, \text { h:y }: X \rightarrow \text { void, } x: X>\text { void }
\end{gathered}
$$

To finish the proof, perform an elimination on $h$ with $x$; so $h(x)$ belongs to void. This happens because rec(t. $t)$ is empty and induction performs a structural analysis on an arbitrary element. But elimination only gives an analysis of some specific element whose pattern can be displayed by applications of the elimination rule. 
The computational form extracted from this rule is a recursive procedure where $h$ represents a recursive call of the procedure. (The procedure is fix $(f, x . f(x))$ as we will see in part III.)

Before presenting the complete rule, we examine another special case which illustrates the treatment of parameters.

$$
\begin{aligned}
& \text { H, w:rec(t,x. T })(a)>>G(a / g) \\
& \text { by ind w over A using g.G at } U_{1} \text { new } X, h \\
& H, X: A \rightarrow U_{\mathfrak{b}} h:(g: A \rightarrow w: X(g) \rightarrow G), \\
& \text { g:A,w:T(X/t,g/x) }>>G
\end{aligned}
$$

It is noteworthy that $\mathrm{X}$ is a new type with parameter over $\mathrm{A}$. Here is the complete rule.

Let $R_{1}=\operatorname{rec}\left(t_{1}, x_{1} . T_{1} ; \ldots ; t_{n}, x_{n} . T_{n} ; t_{1}\right)$ and

let $H^{*}$ be $X_{1}: A_{1} \rightarrow U_{k}, \ldots, X_{n}: A_{n} \rightarrow U_{k}$,

$$
\begin{aligned}
& h_{1}:\left(g_{1}: A_{1} \rightarrow w_{1}: X_{1}\left(g_{1}\right) \rightarrow G_{1}\right), \ldots, \\
& h_{n}:\left(g_{n}: A_{n} \rightarrow w_{n}: X_{n}\left(g_{n}\right) \rightarrow G_{n}\right)
\end{aligned}
$$

Then the general induction rule is:

4. $\quad H, w_{l}: R_{1}(a)>>G_{l}\left(a / g_{1}\right)$

by ind $w_{1}$ over $A_{1}, \ldots, A_{n}$, using $g_{1} \cdot G_{1}, \ldots, g_{n} \cdot G_{n}$

$$
\text { new } X_{1}, \ldots, X_{n}, v_{1}, \ldots, v_{n}, w_{1}, \ldots, w_{n}, h_{b} \ldots, h_{n} \text { at } U_{k}
$$

1. $H, H^{*}, g_{1}: A_{1}, w_{1}: T_{1}\left(X_{j} / t_{j}, g_{1} / x_{1}\right)>>G_{1} \quad\left[E X T e_{1}\right]$

$$
\text { n. } H_{1} H^{*}, g_{n}: A_{n}, w_{n}: T_{n}\left(X_{j} / t_{j}, g_{n} / x_{n}\right)>>G_{n} \quad\left[E X T e_{n}\right]
$$

The extracted term is $f i x\left(h_{1}, g_{1} \cdot e_{1}^{\prime} ; \ldots ; h_{n}, g_{n} \cdot e_{n}^{\prime} ; h_{1}\right)(a)\left(w_{i}\right)$ where $e f$ is $\lambda w_{j} \cdot e_{j}$ with any $X_{k}$ replaced by rec $\left(t_{1}, x_{1}, T_{1} ; \ldots ; t_{n}, ; t_{k}\right)$. It is explained below.

\section{Extraction}

The constructive character of the logic requires that with each rule there be explicit instructions for how to extract its computational content. In the formation rule the content is the conclusion, in the introduction and elimination rules the extraction forms are inherited directly from the rules for building nonrecursive types, as illustrated below. But for the induction rule a new computa- 
tional form is required. The new form must specify how to compute recursively with elements of the recursive type. The key idea for specifying this form is that use of the induction hypothesis of the form $h: x: A \rightarrow y: X(x) \rightarrow G(y / g)$ corresponds to a recursive call. We look first at examples and then explain the specific form.

Let us see how elements of rec(t.int|(int \#t)) are built. Let $\mathrm{T}$ be int|(int\#t)

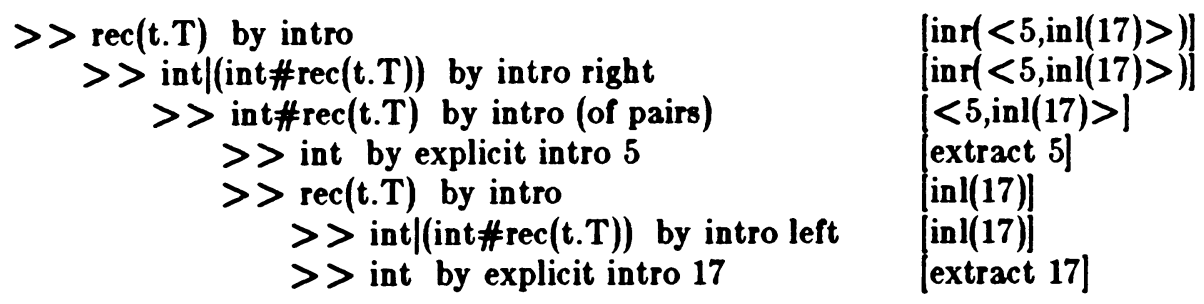

The extracted terms are displayed in square brackets and are constructed bottom-up after the proof is complete. Now when we build elements by induction, we are performing a structural analysis of terms such as $\operatorname{inr}(<5, \operatorname{inl}(17)>)$. Here is an example for $\mathrm{T}$ as above.

x:rec(t.T) $>>$ int by elim $x$ at $U_{1}$ new $X, h$

$X: U_{1}, h: X \rightarrow$ int, $x:($ int|int $\# X)>>$ int by elim $x$ new $x 1, x 2$ [decide(x; x1.x1; x2.h(2of(x2)))]

$X: U_{1}, h: x \rightarrow$ int, $x:($ int $\mid$ int $\# X), x 1:$ int $>>$ int by hyp $x 1$ [x1]

$X: U_{1}, h: X \rightarrow$ int, $x:($ int|int\#X), $x 2:($ int $\# X)>>$ int by elim $x 2$ new $u, v \quad[h(2 \circ(x 2))]$

$X: U_{1}, h: X \rightarrow$ int, $x 2:($ int\# $X)$, u:int, $v: X, x 2=\langle u, v\rangle>>$ int by elim $h[h(v)]$

Again the extracted forms are created bottom-up. In the second from last extracted form, decide( $(x) x 1 . x 1 ; x 2 . h(2 \circ f(x 2)))$, h represents a recursive call, and $20 f(x 2)$ selects the second component of the pair $\langle u, v\rangle$ (it is defined as $\operatorname{spread}(z ; u, v . v)$ ). A reasonable expression for the final extracted form would be

$$
\text { fix(h,x. decide(x; x1.x1; x2.h(2of(x2)))). }
$$

In section III we will present a systematic treatment of such forms.

\section{Equality}

Two recursive types, say rec(t. $\left.T_{1}\right)$ and $\operatorname{rec}\left(t . T_{2}\right)$ are equal when $t: U_{1} \gg T_{1}=T_{2}$ in $U_{1}$. More generally, $\operatorname{rec}\left(t, x . T_{1}\right)(a)=\operatorname{rec}\left(t^{\prime}, x^{\prime} . T_{2}\right)(b)$ when $\lambda x \cdot \lambda t . T_{1}=\lambda x^{\prime} \cdot \lambda t^{\prime} \cdot T_{2}$ in $A \rightarrow U_{1} \rightarrow U_{1}$ and $\lambda t$. $T_{1}(a / x)=\lambda t^{\prime} \cdot T_{2}(b / x)$ in $U_{1}$. For example, if $f(0)=$ int, then $\operatorname{rec}(t, x . x \neq t \mid x)($ int $)=\operatorname{rec}(t, y$. 
$y \# t \mid y)(f(0))$. Also rec(t,x. int\#t|x)(int) $=\operatorname{rec}(t, x . x \# t \mid x)($ int $)$. But notice, we do not equate int\#int and rec(t. int\#int). The general rule is:

5. $\quad H>>\operatorname{rec}\left(t_{1}, x_{1} . T_{1} ; \ldots ; t_{n}, x_{n} . T_{n} ; t_{1}\right)(a)=$

$$
\operatorname{rec}\left(t_{1}, x_{1} \cdot T_{1} ; \ldots ; t_{m}^{\prime}, x_{m}^{\prime}, T_{m}^{\prime} ; t_{j}\right)(b) \text { in } U_{1}
$$

by equality over $A_{1}, \ldots, A_{n}$

(provided $\mathrm{n}=\mathrm{m}$ and $\mathrm{i}=\mathrm{j}$ )

1. $H, x_{1}: A_{1}, t_{1}: A_{1} \rightarrow U_{k}, \ldots, t_{n}: A_{n} \rightarrow U_{k} \gg T_{1}=T_{1}\left(t_{j} / t_{\jmath}, x_{1} / x_{1}\right)$ in $U_{1}$

n. $H_{, x_{n}}: A_{n}, t_{1}: A_{1} \rightarrow U_{k}, \ldots, t_{n}: A_{n} \rightarrow U_{k} \gg T_{m}=T_{m}^{\prime}\left(t_{\jmath} / t_{\jmath}, x_{1} / x_{1}\right)$ in $U_{1}$ $n+1 . H>a=b$ in $A$

Propositions-As-Types

In theories which adapt the propositions-as-types principle, such as Nuprl, AUTOMATH, Martin-Lör's theories, Girard's theories [21,46], etc., recursive definitions of types provide recursive definitions of propositions as well. Thus in such theories we have a version of infinitary logic. We rely on this feature to define the domains of partial recursive functions in section 2.2 . The important point is that equalities such as ( $x=1$ in int) can be treated as types in recursive definitions.

\section{Representing Well-Founded Trees}

A recursive type such as $\operatorname{rec}(w . x: A \#(B(x) \rightarrow w))$ represents the W-type of well-founded trees used to represent Brouwer ordinals [6,46]. Martin-Lör's rules for the W-type can be derived from rules for recursive types. For example his introduction rule is

$$
\frac{a \in A \quad f \in B(a) \rightarrow W}{\sup (a, f) \in W x \in A . B(x)}
$$

which corresponds to

$$
\begin{aligned}
H>> & <a, f>\text { in } \operatorname{rec}(w . x: A \# B(x) \rightarrow w) \\
H> & ><a, f>\text { in } x: A \# B(x) \rightarrow w \\
H & >\text { a in } A \\
H & >>\text { in } B(a) \rightarrow \operatorname{rec}(w . x: A \# B(x) \rightarrow w) .
\end{aligned}
$$

The induction rule for rec(w. $x: A \# B(x) \rightarrow w)$ corresponds to Martin-Lör's elimination rule. Essentially this is Martin-Lör's rule in our notation 
$H, x: W y \in A . B(y)>>G$

$H, y: A, f: B(y) \rightarrow W y \in A . B(y)$,

$z:(v: B(y) \rightarrow G(f(v) / x))>>G$

$[\operatorname{ext} d(y, f, z)]$

The extracted term is a tree induction form, let us call it $\operatorname{tind}(\mathbf{x} ; \mathbf{y}, \mathbf{f}, \mathbf{z} . \mathrm{d})$. It satisfies the computation rule tind $(\sup (\mathbf{a}, \mathrm{b}) ; \mathbf{y}, \mathbf{f}, \mathbf{z . d})=\mathrm{d}(\mathrm{a} / \mathrm{y}, \mathrm{b} / \mathbf{f}, \lambda \mathbf{v} \cdot \operatorname{tind}(\mathbf{f}(\mathbf{v}) ; \mathbf{y}, \mathbf{f}, \mathbf{z . d}) / \mathbf{z})$.

This rule can be seen as a special way of using the induction rule as follows.

$H, x: \operatorname{rec}(w \cdot y: A \#(B(y) \rightarrow w))>>G$

1. $H, X: U_{b}, h:(x: X \rightarrow G), x: y A \#(B(y) \rightarrow X)>>G$

1.1. $H, X: U_{b} h:(x: X \rightarrow G), y: A, f: B(y) \rightarrow X>>G$

1.1.1. H, $X: U_{b}, h:(x: X \rightarrow G), y: A, f: B(y) \rightarrow X>>v: B(y \rightarrow G(f(v)(x)$

1.1.2. $H, X: U_{1}, h:(x: X \rightarrow G), y: A, f: B(y) \rightarrow X, z:(v: B(y) \rightarrow G(f(v) / x))>>G$ by induction at $U_{i}$, new $X, y, h$ by elim $x$

by seq $v: B(y) \rightarrow G(f(v) / x)$ new 3 by explicit intro $\lambda v . h(f(v))$ [ext d(y, $\mathbf{f}, \mathbf{z})$ ]

Now define $g(x, h)=d(\operatorname{lof}(x), 2 o f(x), \lambda v \cdot h(2 \circ f(x)(\operatorname{lof}(x))))$ where $\operatorname{lof}(x)=\operatorname{spread}(x ; u, v . u), 20 f(x)=$ $\operatorname{spread}(x ; u, v . v)$. Then $\lambda x . \operatorname{tind}(x ; y, f, z . d(y, f, z))$ corresponds to $f(x)(h, x . g(x, h))$. We see that $\operatorname{fix}(\mathbf{h}, \mathbf{x} \cdot \mathbf{g}(\mathbf{x}, \mathbf{h}))(<\mathrm{a}, \mathrm{b}>)=\mathrm{d}(\mathbf{a}, \mathrm{b}, \lambda \mathbf{v} \cdot \mathbf{h}(\mathbf{b}(\mathbf{v})))=\operatorname{tind}(\sup (\mathbf{a}, \mathrm{b}) ; \mathbf{y}, \mathbf{f}, \mathbf{z} \cdot \mathrm{d}(\mathbf{y}, \mathbf{f}, \mathbf{z}))$.

Example

As a nontrivial example of mutually recursive types, consider the definitions of when a type variable is "positive" or "negative" in a type expression (this notion of positive will be a generalization of what we use in the theory). Here "type expressions" are terms built up from type variables using " $\rightarrow$ ". The definitions are:

Type variable $v$ is positive in type expression $t$ iff $t$ is a type variable, or $t$ is $t_{1} \rightarrow t_{2}, v$ is negative in $t_{1}$ and positive in $t_{2}$.

Type variable $v$ is negative in type expression $t$ iff $t$ is a type variable other than $v$, or $t$ is $t_{1} \rightarrow t_{2}, v$ is positive in $t_{1}$ and negative in $t_{2}$.

To represent type expressions as a datatype in the theory we define

Type_var $=$ atom

Type_exp $=$ rec(T.Type_var $\mid \mathrm{T} \# \mathrm{~T})$

then the definition of positive (with respect to $v$ ) is

$\operatorname{Pos}(v)={ }_{d} \operatorname{rec}\left(P, t\right.$. decide(t; a.true; q.spread $\left(q ; t_{1}, t_{2} \cdot N\left(t_{1}\right) \# P\left(t_{2}\right)\right) ;$

$N, t$. decide $\left(t ; a . \neg a=v\right.$ in atom; q.spread $\left.\left(q ; t_{1}, t_{2} \cdot P\left(t_{1}\right) \# N\left(t_{2}\right)\right) ; P\right)$

and the definition of negative is the same, with $\mathrm{N}$ selected. The following statement, asserting Posi- 
tive and Negative are decidable propositions, can be proved by induction on $t$.

$$
>\text { t:Type_exp } \rightarrow \mathrm{v}: T y p e \_v a r \rightarrow(\operatorname{Pos}(v)(t) \vee \neg \operatorname{Pos}(v)(t)) \# \operatorname{Neg}(v)(t) \vee \neg \operatorname{Neg}(v)(t)
$$

where in general $\neg \mathrm{A}$ means $\mathrm{A} \rightarrow$ void.

\section{Recursive functions}

\section{Background}

For each recursive data type in the theory, there is a computation form associated with its elimination rule. For example, natural number induction provides a form written ind(e;b;u,v.h) in Nuprl, and it satisfies the computation rule.

$$
\begin{aligned}
& \operatorname{ind}(0 ; b ; u, v . h)=b \\
& \operatorname{ind}(n ; b ; u, v . h)=h(n / u, i n d(n-1 ; b ; u, v . h)(v) .
\end{aligned}
$$

This is recognizable as a form of primitive recursion with values in any type. As a special case we have the ordinary primitive recursion scheme for defining functions into the natural numbers, e.g. factorial(n) is

$$
\operatorname{ind}(\mathbf{n} ; \mathbf{1} ; \mathbf{u}, \mathbf{v} . \mathbf{u} * \mathbf{v})
$$

Likewise list induction gives rise to a form of primitive recursion on lists. We have also seen that the defined recursive types provide a recursive computation form. We have written them as fix $(f, x . F)$. We have not discussed these forms in detail yet, but it is clear that for any defined recursive type, say rec(t,x. T), the extract form of the induction rule, say fix $(f, x . F)$, is a well defined computable function whose domain is $\operatorname{rec}(t, x . T)$.

One might ask whether "every recursive function" in some sense can be obtained in this way. The answer is "no" in the following sense. We can say precisely what we mean by "every recursive function." Namely, we understand from Basic Recursive Function Theory (BRFT) [21] the concept of an algorithm and a partial recursive function. We can show that the forms needed to extract from induction rules on recursive types are a proper subset of the collection of all algorithms. In particular none of the recursively defined types can explicitly mention the extracted function in their own definition. Also because we restrict the formation of recursive types to positive forms, not "all algorithms" are represented in extractions. Moreover, even if every algorithm could be represented by an 
induction form, it is not clear that they can be collected into a type in a natural way.

In order to represent "all algorithms" in the theory, we turn to the insights from Herbrand, Gödel, Church, Kleene and Turing who first tried to formalize the concept. Herbrand's ideas are particularly suitable because they arose in the context of constructive mathematics and logic (see [49]). (They were also the first expression of the concept of algorithm as we now know it.)

Herbrand realized that we could understand a rule and treat it as a precise concept before we understood exactly what the domain of application was. For us this means we can use the rule to define its domain of definition. That domain will depend on what we can prove about the rule, but even without an abstract idea of proof we know how to execute the rule.

One way to develop Herbrand's idea in type theory is to define an internal notion of a rule, for example to define Turing machines or mu-recursive functions as inductive classes of functions. This approach is illustrated in [16]. Another approach is to introduce partial functions into the very formalization of constructive mathematics itself. For example, this is done classically in LCF [22] and by Kleene [26] for number theory. We follow this later path, taking partial functions as basic concepts. We introduce new canonical forms to denote partial functions, written fix $(f, x . F)$ in the style of ML. The computation rule for these forms is $f i x(f, x . F)(a)=F(a / x, f i x(f, x$. $F) / f)$. Based on this rule we know how to say that a fix form converges on an input. We could express the domain of convergence of fix $(f, x . F)$ mapping $A$ to $B$ as $\{y: A \mid f i x(f, x . F)[y]$ in $B\}$. It would be possible to prove fix $(f, x . F)|a|$ in $B$ for specific a by a series of reduction steps. For example, we can prove fix(f,x. inteq $(x ; 0 ; 1 ; f(x-1)))[0]=1$ in int by one reduction step. We might prove some instances of fix $(f, x . F)[y]$ in $B$ by induction. But a serious disadvantage to this simple approach is that we cannot reason about $\{y: A \mid f i x(f, x . F)[y]$ in $B\}$ inductively although it posses an inductive structure. We might in fact be able to show that for any element of this domain fix $(f, x . F)[y]=b$ in $B$ if we had access ot the inductive structure. We will see a particular example of this below for the " $3 x+1$ function."

In order to provide access to the inductive structure of the domain of partial functions, we introduce a new (noncanonical) form into the theory, $\operatorname{dom}(f)(g)$. The type $\operatorname{dom}(f i x(f, x . F))(a)$ expresses the concept that fix $(f, x . F)[a]$ converges on input a from type $A$. The rules are arranged so that 
when $\operatorname{dom}(\operatorname{fix}(f, x . F))(a)$ is true for $a$ in $A$ and when $F(f i x(f, x . F) / f, a / x)$ is functional in $B$, then fix $(f, x . F)$ is a partial function from $A$ to $B$ which converges on all a for which $\operatorname{dom}(f i x(f, x . F))(a)$ is true.

It has proven to be useful in programming and in the theory of algorithms to think in terms of the space of all partial functions from A to B. To define this concept in type theory, we introduce the new constructor, $\mathrm{x}: \mathrm{A} \sim>\mathrm{B}$, denoting the partial function space. The elements of this space are named by expressions which reduce to the fix $(f, x . F)$ canonical forms and which are defined by the introduction and equality rules for the $\mathrm{x}: \mathrm{A} \sim>\mathrm{B}$ type. The precise rules are given below.

\section{Domain Predicates}

Syntax For Algorithms

The representation of algorithms that we use is based on the conception that the computation proceeds by successively evaluating a functional and follows closely the style of PP $\lambda$ of Edinburgh LCF [22]. (In part this decision was made because the ML part of LCF is a component of Nuprl.) We write fix $\left(f_{1}, x_{1}, F_{1} ; \ldots ; f_{n}, x_{n} . F_{n} ; f_{1}\right)$ where $F_{J}$ is a well-defined expression which may contain occurrences of $f_{1}$. We say that occurrences of $f_{1}$ and $x_{1}$ in $F_{j}$ are bound. To minimize the notational complexity, we present in this section the details only for the single function case, fix(f,x. F). To simplify the treatment of domains, we do not allow in $F$ any form $\lambda x$.b where $b$ contains occurrences of $f$. Any such form can be replaced by fix $(g, x . b)$ where $g$ does not occur in $b$, and the result will be an equivalent function.

\section{Examples of Domain Predicates}

One of the computation forms in Nuprl is inteq $\left(e ; e_{2} ; t_{1} ; t_{2}\right)$ which evaluates to $t_{1}$ if $e_{1}=e_{2}$ and to $t_{2}$ otherwise where $e_{1}$ and $e_{2}$ are integer expressions. The well-known $3 x+1$ function can be written in terms of inteq and a modulus function $\bmod (x, y), \operatorname{notice} \bmod (x, 2)=0$ iff $x$ is even. The function is:

$$
\text { fix }(f, x . \text { inteq }(x ; 1 ; 0 ; \text { inteq }(\bmod (x, 2) ; 0 ; f[x / 2] ; f[3 * x+1])))
$$

Call the function $g$. The domain on which $g$ converges is the set of natural numbers $x$ such that $x=1$ or $x>1, x$ is even and $x / 2$ is in the domain or $x>1, x$ is odd and $3 * x+1$ is in the domain. 
This is defined in the theory as

$$
\begin{aligned}
& \operatorname{rec}(D, x .(x=1 \text { in int }) \mid x>1 \&(\bmod (x, 2)=0 \text { in int }) \& D(x / 2) \mid \\
& x>1 \& \neg(\bmod (x, 2)=0 \text { in int }) \& D(3 * x+1)) \text {. }
\end{aligned}
$$

Recall from 1.3 that $(x=0$ in int $),(\bmod (x, 2)=0$ in int $)$ and $x>1$ can be construed as types. The $\&$ operator is just another symbol for \# which is used when its operands are thought of as propositions. A more direct translation of the function yields the equivalent recursive type.

$$
\operatorname{rec}(D, x \text {. inteq }(x ; 1 ; \operatorname{true} ; \operatorname{inteq}(\bmod (x, 2) ; 0 ; D(x / 2) ; D(3 * x+1))))
$$

where true is a one element type. The idea is that the recursive type is nonempty exactly when the proposition it expresses holds.

\section{Pattern of Definition}

To express the relationship between the recursive function $g$ and its domain, we introduce into the theory a new (noncanonical) expression written $\operatorname{dom}(\mathbf{g})(\mathbf{x})$; thus dom is a new form in the theory. We must also introduce into the metatheory a procedure for computing the recursive form of $\operatorname{dom}(g)(x)$ for any recursive function $g$. We denote this algorithm by $D$; its result on inputs $g$ and $x$ is $\mathrm{D}(\mathrm{g}, \mathbf{x})$. By definition $\operatorname{dom}(\mathrm{g})(\mathbf{x})=\mathrm{D}(\mathrm{g}, \mathrm{x})$. The expression $\mathrm{D}(\mathrm{g}, \mathbf{x})$ is not a new form in the theory, it is provided only so that $\operatorname{dom}(\mathbf{g})(\mathbf{x})$ can be properly defined. $\mathrm{D}(\mathbf{g}, \mathbf{x})$ is computed under the assumption that arguments to a function are evaluated before the function is applied. This means it will compute an empty domain for certain functions that would have a nonempty domain under lazy evaluation. But the advantage is that type information is not needed in $\operatorname{dom}(\mathrm{g})(\mathrm{x})$. In the course of defining $D(g, x)$ we also need an auxiliary function $C(g)$.

We will need an environment during the computation of $D(g, x)$. The actual function we describe is $D(g, x, E)$ where $E$ is an environment keeping track of which type variables are being defined recursively.

An environment $E$ is function f:identifiers $\rightarrow$ expressions, thought of as a list of identifier/expression pairs. The expressions needed are partial function definitions. The only operations needed on environments are applications, $E(u)$, to look up a value, and additions. An addition is written E[u:e] which means extended the (finite) function to include the pair $\langle u, e\rangle$; so that 
$E[\mathbf{u}: e](\mathbf{u})=e$.

The expression $\mathrm{D}(\mathrm{g}, \mathrm{x}, \mathrm{E})$ will denote $\operatorname{dom}(\mathrm{g})(\mathbf{x})$ in environment $\mathrm{E}$. The first argument, $\mathrm{g}$, will always denote a partial function, either in canonical form, as fix $(f, x . F)$, or in elimination form as in ind $(e ; b ; u, v . F)$. In the course of defining $D(g, x, E)$, we will need to specify the conditions under which an expression $F$ in environment $E$ is well-defined. We denote these as $\mathrm{C}(\mathrm{F}, \mathrm{E})$, for example $C\left(<F_{1}, F_{2}>, E\right)$ is just $C\left(F_{1}, E\right) \# C\left(F_{2}, E\right)$. The definition of $C(F, E)$ is given simultaneously with that for $D(g, x, E)$. The concepts are mutually recursive; for instance $D(f i x(f, x . F), y, E)=\operatorname{rec}(f, x . C(F, E))(y)$ and $C(g \mid e], E)=C(g, E) \# C(e, E) \# D(g, e, E)$. We read $D(f i x(f, x . F), y, E)=\operatorname{rec}(f, x . C(F, E))(y)$ to mean that the domain of $f(x)(f, F)$ is a recursive type with internal name $f$. For convenience $f$ is used to denote the type form, $\operatorname{dom}(\boldsymbol{f i x}(f, x . F))$, as well as the function (a special operation, $*(u, E)$, will be used to replace an occurrence of $f$ as a domain type by its occurrence as a function). This recursive type says that the domain condition is met by guaranteeing that $F$ is well-defined. Here now is a complete definition of $\mathrm{D}(\mathrm{g}, \mathrm{x}, \mathrm{E})$ and $\mathrm{C}(\mathrm{F}, \mathrm{E})$. We take $\mathrm{D}(\mathrm{g}, \mathrm{x})=\mathrm{D}(\mathbf{g}, \mathbf{x}, \mathbf{n i l})$.

\section{Definition:}

$D(f i x(f, x . F), u, E)=\operatorname{rec}(f, x . C(F, E[f: f i x(f, x . F)]))(u)$

$D\left(\operatorname{spread}\left(p ; a, b . F^{\prime}\right), u, E\right)=\operatorname{spread}\left(*(p, E) ; a, b . D\left(F^{\prime}, u, E\right)\right)$

decide, ind, list-ind, int-eq, atom-eq, less are similar.

If $h$ is a variable, then

if $\mathrm{E}(\mathrm{h})=\mathbf{f i x}(\mathbf{h}, \mathbf{z} . \mathrm{H})$

then $D(h, u, E)=h(u)$ since $h$ is the function whose domain we are

computing and we have agreed to use $h$ as the name of the domain

$$
\left.\left.D\left(t \mid t^{\prime}\right], \mathbf{u}, E\right)=D\left(t, t^{\prime}, E\right) \# \operatorname{dom}\left(t \mid t^{\prime}\right]\right)(\mathbf{u})
$$

Now the definition of $\mathrm{C}(\mathrm{F}, \mathrm{E})$

$$
\mathrm{C}\left(<\mathrm{F}_{1}, \mathrm{~F}_{2}>\mathrm{E}\right)=\mathrm{C}\left(\mathrm{F}_{1}, \mathrm{E}\right) \# \mathrm{C}\left(\mathrm{F}_{2}, \mathrm{E}\right)
$$

likewise for the other intro forms: inl, inr, $\lambda x . F, \ldots$

$$
\begin{aligned}
& C\left(\lambda x . F_{1}, E\right)=C\left(F_{1}, E\right)^{\dagger} \\
& C\left(\operatorname{inl}\left(F_{1}\right), E\right)=C\left(F_{1}, E\right)
\end{aligned}
$$

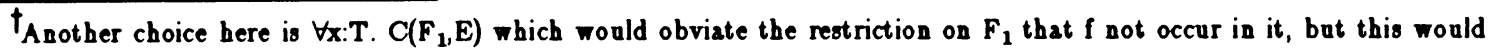
require keeping track of the type of subterms in order to know that $x$ has type $T$.
} 


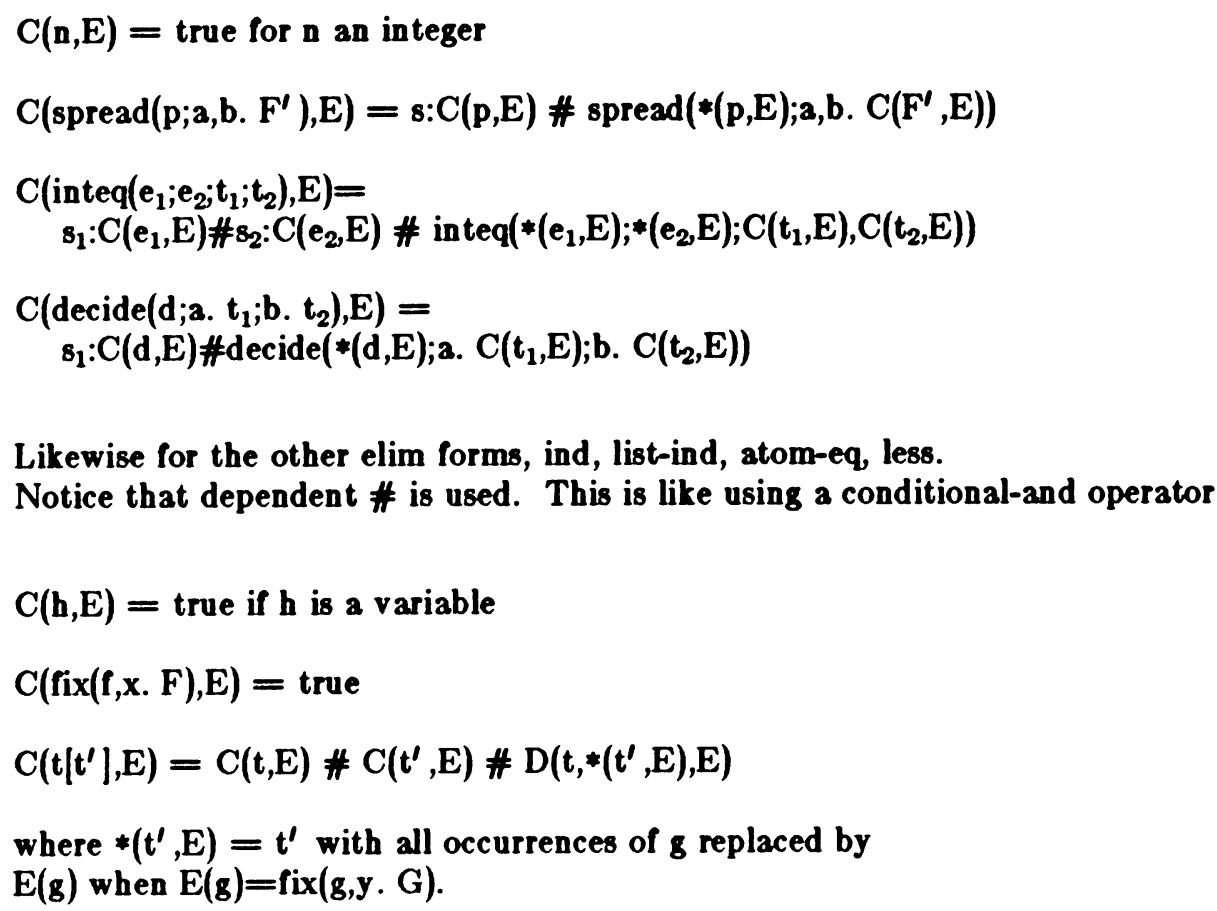

This ends the definition.

These rules define $\operatorname{dom}(f)(x)$ for any partial function (or algorithm) as a recursive type. Thus we can use the rules for recursive types to prove propositions of the form $\operatorname{dom}(f)(a)$ or $\forall x: A . d o m(f)(x)$ and so forth. We might have tried to assert that fix $(f, x, F)$ is defined at a by proving the wellformedness assertion fix $(f, x . F)[a]$ in $B$. We could even use this technique to prove properties of those values for which fix $(f, x . F)[y]$ in $B$ is true. But without $\operatorname{dom}(f i x(f, x . F))$ we cannot prove properties of the domain by induction.

\subsection{Rules}

A partial function $f$ from $A$ into $B$ actually maps $\{x: A \mid \operatorname{dom}(f)(x)\}$ into $B$. By this notation we indicate that the domain information is not needed for computing the value of $f$, only for insuring that the computation is sensible. This is because the set notation $\{x: A \mid B\}$ in Nuprl denotes those elements of $A$ such that $B(a / x)$ is true (but unlike in Martin-Lör's theories, the proof information, $b \in B(a / x)$ is not included with the elements). We see these ideas in the elimination rule for partial functions, there the argument to $f$ is simply a, but one of the subgoals requires the proof $\operatorname{dom}(f)(a)$. 
It is convenient to collect these functions into a single type, such a collection is commonly and easily defined in set theory for instance. This type is essentially the union of the types $\{x: A\}$ $\operatorname{dom}(f)(x)\} \rightarrow B$ for all algorithms $f$. There are no union types as such in type theory, so this concept is represented by a new constructor written $\mathrm{A} \sim>\mathrm{B}$. The rules for $\mathrm{A} \sim>\mathrm{B}$ fall into these categories: formation, introduction, elimination, equality, and computation.

\section{Formation Rule}

$\mathrm{H}>>\mathrm{x}: \mathrm{A} \sim>\mathrm{B}$ in $\mathrm{U}_{1}$

1. $\mathrm{H}>>\mathrm{A}$ in $\mathrm{U}_{\mathrm{i}}$

2. $\mathrm{H}, \mathrm{x}: \mathrm{A}>\mathrm{B}$ in $\mathrm{U}_{1}$

Introduction Rule

$\mathrm{H}>>\mathrm{x}: \mathrm{A} \sim>\mathrm{B}$ by intro at $\mathrm{U}_{\mathrm{l}}$ new $\mathrm{f}, \mathrm{y}$ [EXT fix $\left.(\mathrm{f}, \mathrm{y} . \mathrm{F})\right]$

1. $H, f:(x: A \sim>B), y: A>B(y / x)$ [EXT F] (primed subgoals do not apply for this first subgoal)

2. $\mathrm{H} \gg \mathrm{x}: \mathrm{A} \sim>\mathrm{B}$ in $\mathrm{U}_{\mathrm{I}}$

In the proof of $B(y / x)$ we do not want to require that uses of $f$ satisfy the domain condition because the domain condition is defined in terms of the proof being built at this point. That is, if the extracted object is $F$, then $\operatorname{dom}(f)(z)$ will be $D(f i x(f, y . F), z)$.

Explicit Introduction Rule

$H>>$ fix $(f, y . F)$ in $x: A \sim>B$ by intro at $U_{1}$ new $y$

1. $H, f:(x: A \sim>B), A>F$ in $B(y / x)$ (primed subgoals do not apply for this first subgoal)

2. $\mathrm{H}>>\mathrm{x}: \mathrm{A} \sim>\mathrm{B}$ in $\mathrm{U}_{1}$

\section{Elimination}

$\mathrm{H}, \mathrm{f}:(\mathrm{x}: \mathrm{A} \sim>\mathrm{B}), \mathrm{H}^{\prime}>>\mathrm{G}$ by elim $\mathrm{f}$ on a

1. $H, f:(x: A \sim>B), H^{\prime}>>a$ in $A$

2. $H, f:(x: A \sim>B), H^{\prime}>>\operatorname{dom}(f)(a)$

3. $\mathrm{H}, \mathrm{f}:(\mathrm{x}: \mathrm{A} \sim>\mathrm{B}), \mathrm{H}^{\prime}, \mathrm{y}: \mathrm{B}(\mathrm{a} / \mathrm{x}), \mathrm{y}=\mathrm{f}[\mathrm{a} \mid$ in $\mathrm{B}(\mathrm{a} / \mathrm{x})>>\mathrm{G}$

\section{Equality}

$H>>f|a|$ in $B(a / x)$ by intro using $x: A \sim>B$
1. $H>>$ in $(x: A \sim>B)$
2. $H>>a$ in $A$
3'. $\mathrm{H}>>\operatorname{dom}(\mathrm{f})(\mathrm{a})$ 
$H \gg \operatorname{fix}(f, x . F)=f i x(g, y . G)$ in $x: A \sim>B$

1. $H>>\operatorname{fix}(f, x . F)$ in $x: A \sim>B$

2. $H, x: A, f:(x: A \sim>B)>>F=G(g / f, x / y)$ in $B$

$H>>\operatorname{fix}(f, x . F)[a]=f i x(g, y . G)[b]$ in $B$

$H>>a=b$ in $A$

$H>\operatorname{fix}(f, x . F)=f i x(g, y . G)$ in $x: A \sim>B$

$H>>f i x(f, x . F)[a]=F(a / x, f i x(f, x . F) / f)$ in $B(a / x)$ by computation using $x: A \sim>B$

1. $H>>f i x(f, x . F)$ in $x: A \sim>B$

2. $H>>a$ in $A$

3. $H>>\operatorname{dom}(\operatorname{fix}(f, x . F))(a)$

Among the computation rules, we allow the following reduction.

Computation

$f i x(f, x . F)[a] \rightarrow F(a / x, f i x(f, x . F))$

\subsection{Recursive Types Over Partial Function Spaces}

The main difficulty with defining types like rec $(t . t \rightarrow t)$ is that they allow nonterminating functions as members. It seems plausible that this difficulty could be avoided by using the partial function space constructor instead. To this end we allow without restriction the $\sim>$ operator in recursive type definitions. Thus $\operatorname{rec}(t . t \sim>t)$ is a legitimate type. Let us derive an element of this type.

Let $\mathrm{R}$ denote $\operatorname{rec}(\mathrm{t} . \mathrm{t} \sim>\mathrm{t})$

$>>$ fix(f. $f(f))$ in $R$ by rec intro

$f: R \quad>P f[]$ in $R$ by $R$ elim on $f$

$f: R \sim P R \quad P>f[f]$ in $R$ by $f$ elim

1. $\mathrm{f}: \mathrm{R} \sim \mathrm{P} P \mathrm{P}$ in $\mathrm{R}$ by $\mathrm{R}$ - intro $f: R \sim P R \quad>P$ in $R \sim>R$ by hyp.

2. $f: R \sim>R \quad>f$ in $R \sim>R$ by hyp.

Notice that if the primed subgoals were in force for the f elim step, we would be required to prove $\operatorname{dom}(f)(f)$. But this is not required for well-formedness.

What is the domain of fix $(f, f[f])$ ? A calculation shows this: 
$\operatorname{dom}(\operatorname{fix}(f . f(f)))(a)=D(f i x(f . f(f)), a, \lambda x . \$)=$

$\operatorname{rec}(f . C(f[f], \lambda x .[f: f i x(f . f[f])]))(a))=$

$\operatorname{rec}(\mathrm{f} . \mathrm{C}(\mathrm{f}, \ldots$ _ $\# \mathrm{C}(\mathrm{f}, \ldots) \# \mathrm{D}(\mathrm{f}, \mathrm{fix}(\mathrm{f} \mathrm{f}[\mathrm{f}], \lambda \mathbf{x} .[\mathrm{f}: \mathrm{fix}(\mathrm{f} . \mathrm{f}[\mathrm{f}])]))=$

rec(f. true\#true\#dom(f)(fix(f. f(f])))(a).

The total elements of $\operatorname{rec}(t . t \sim>t)$ are defined by $\{f: \operatorname{rec}(t . t \sim>t) \mid \forall x: \operatorname{rec}(t . t \sim>t)$. $\operatorname{dom}(\mathbf{f})(\mathbf{x})\}$

\section{Foundational Issues}

\subsection{Constructive Meanins}

How do we know that this theory is sensible and consistent? One approach to the question is a proof-theoretic reduction of this formal theory to a formally simpler one believed to be consistent and meaningful, say to Martin-Lör's Intuitionistic Type Theory [31] or to the theory of species [46] or to Nuprl without recursive types. For instance, in the case of Nuprl without types, R. Harper [23] has provided a reduction to a technically simpler theory similar to Intuitionistic Type Theory [31].

Another possibility is to prove the consistency of this theory directly, say as Tait [48] did for Gödel's theory of functionals [46]. This would involve appeal to some principles of greater proof theoretic strength. But Martin-Löf has analyzed the proof methods in a Tait style proof, and the result is his doctrine of canonical forms [31]. It is possible to appeal directly to this doctrine to confirm that a theory is sensible. In a similar way, it is possible to appeal to a basic constructive theory of inductive definitions to explain the meaning of the formal theory. Stuart Allen [1] has given such an analysis for Nuprl.

For this theory we have not provided a proof-theoretic reduction to a simpler theory, although it is plausible that the notion of a W-type in Intuitionistic Type Theory would support the concept of our recursive type, and it is plausible that partial functions could be represented by extending the methods of Constable [12]. For the concept of recursive types over \# and |only, there is a reduction to the types of Nuprl which we sketch in the next subsection.

The account of the meaning of this theory that we have provided by appeal to Nuprl concepts taken along with an explanation of Nuprl itself in terms of fundamental constructive concepts (see 
$[1,13,23])$ is intended to justify the theory. In the present context these foundational ideas may not be sufficiently manifest, but we hope to remedy any deficiencies in this regard by further writings which offer basic explanation of not only these recursive types and partial functions, but also of the more delicate case of recursive types permitting infinite objects (see "Infinite Objects in Constructive Type Theory" forthcoming from us).

\subsection{Reducins Recursive Types Over \# and / to Iterative Types}

In Martin-Lör's type theory [M-L82], it is possible to define types using the induction form. For example, let $f$ be a function from the natural numbers, $N$, into types defined by $f(0)=A$, $f(n+1)=A \# f(n)$. Then the type $\Sigma x \in N$. $f(x)$ collects $A, A \# A, A \# A \# A, \ldots$, and can be thought of as the type of $A$ lists. We call types formed this way iterative types. In this case $x: N \# f(x)$ is an iterative definition of the recursive type rec(f. A\#f).

The iterative type $x: A \# f(x)$ is not as convenient as rec(f. $A \# f)$ because to build an element, one must first decide how long it is, say 3 , and then build $\left\langle 3,<a_{1},\left\langle a_{2}, a_{3}<a\right\rangle>>\right.$. Nevertheless, using iterative types we can build a model of the recursive types involving only \# and | in a simpler theory and thereby establish the consistency of our extension.

The general idea is that the recursive type $\operatorname{rec}(t, x . T)(a)$ will be translated as $x: N \# \hat{T}(a)(x)$ where $\hat{\mathrm{T}}(\mathrm{a})(\mathrm{x})$ is ind(x;void;u,v. $\mathrm{T}(\mathrm{v} / \mathrm{t}, \mathrm{a} / \mathrm{x}))$ where void is the empty type. The elements of the type will essentially include $\langle n, r\rangle$ where $r$ is an element of $\operatorname{rec}(t, x$. $T)(a)$.

Given the translation of partial functions defined above, we can now translate the partial function space $A \sim>B$ into the type $\Sigma P: A \# B \rightarrow U_{1} \cdot(\Sigma x: A . \exists y: B . P(x, y) \rightarrow B)$. This raises the universe level of the concept but provides a consistent interpretation, namely fix $(f, x . F)$ is translated to $<\lambda x$. $G_{F}(x), g\left(G_{F}\right)>$.

It is quite clear from this translation that the fix $(f, x . F)$ notation is much more convenient and that it leads to more efficient computation. In fact, the reliance on proof components is contrary to the character of ordinary mathematical practice. Nevertheless, it does not lead to more expressive power. 


\section{Acknowledgemento}

We thank Stuart Allen for numerous comments and suggestions at every level from notation to philosophy which helped us present these ideas. We also thank Jim Hook for his thoughtful advice and Donette Isenbarger for preparing the manuscript.

\section{References}

[1] Allen, Stuart. The Logical Foundations of Type Theory (to appear).

[2] Aczel, P. An introduction to inductive definitions. Handbook of Mathematical Logic, Barwise, J. (ed.), North Holland, NY (1977) 739-782.

[3] Bates, J.L. and Constable, R.L. Proofs as programs. Tech. Rep., Dept. of Computer Science, Cornell University, TR 82-530, 1982. Also to appear in TOPLAS.

[4] Bishop, E., Foundations of Constructive Analysis. McGraw Hill, New York, NY, 1967. 370 pp.

[5] Boyer, R.S. and Moore, J.S. A Computational Logic. Academic Press, New York, NY, 1979. 397 pp.

[6] Brouwer, L.E.J. Collected Works, Vol. 1, A. Heyting, (Ed.), North-Holland, 1975.

[7] Cartwright, R. User-defined data types as an aid to verifying Lisp programs. Proc. of the Brd Int'l. Collog. on Automata, Languages and Programming, [Michaelson, S. and Milner, R., eds.], Edinburgh University Press, Edinburgh (1976) 228-256.

[8] Cartwright, R. Toward a logical theory of program data. In Logics of Programs, Lecture Notes in Computer Science 131, Springer-Verlag, New York, NY (1982) 37-51.

[9] Church, A. A formulation of the simple theory of types. J. Symbolic Logic, 5, (1940), 56-68.

[10] Constable, Robert L. Constructive mathematics and automatic program writers. In Proc. of IFIP Congres8, Ljubljana, 1971, 229-233.

[11] Constable, Robert L. Constructive mathematics as a programming logic I: some principles of theory. Technical report TR83-554, Dept. of Computer Science, Cornell University, May 1983. (To appear in Proc. of FCT Conf., Springer-Verlag, 1983).

[12] Constable, Robert L. Partial functions in constructive formal theories. In Proc. of 6th G.I. Conference, Lecture Notes in Computer Science 45, Springer-Verlag, New York, NY, 1983.

[13] Constable, R.L., and Bates, J.L. The nearly ultimate PRL. Tech. Rep., Dept. of Computer Science, Cornell University, TR 83-551, 1984.

[14] Constable, Robert L. and O'Donnell, M.J. A Programming Logic. Winthrop, Cambridge, 1978.

[15] Constable, Robert L. and Zlatin, D.R. The type theory of PL/CV3. ACM Trans. on Prog. Lang. \& Syst., 6:1 (Jan. 1984) 94-117.

[16] Constable, Robert L. Mathematics as programming. In Proc. of Workshop on Logics of Programs, Lecture Notes in Computer Science 164, Springer-Verlag, New York, NY, 1983, 116-128.

[17] Curry, H.B., Hindley, J.R. and Seldin, J.P. Combinatory Logic, Volume II. North-Holland Publ. Co., Amsterdam, 1972.

[18] deBruijn, N.G. A survey of the project AUTOMATH. In To H.B. Curry: Essays on Combinatory Logic, Lambda Calculus and Formalism, J.P. Seldin and J.R. Hindley (Eds.), Academic Press, New York, NY, 1980, 579-607.

[19] Demers, A.J. and Donahue, J. Revised report on Russell. Tech. Rep., Department of Computer Science, Cornell University, TR 72389, September 1979. 
[20] Feferman, S. Formal theories for transfinite iterations of generalized inductive definitions and some subsystems of analysis, Intuitionism and Proof Theory, Kino, A., Myhill, J., and Vesley, R.E. (eds.), North Holland, Amsterdam (1970) 303-326.

[21] Girard, J.-Y. Interpretation Fonctionelle et Elimination des Coupures de l'arithmeétique d'ordre supérieur. Ph.D. Thesis, Univ. of Paris VII, 1972.

[22] Gordon, M., Milner, R., and Wadsworth, C. Edinburgh LCF: A Mechanized Logic of Computation, Lecture Notes in Computer Science 78, Springer-Verlag, 1979.

[23] Harper, R. On formalizing and implementing type theory. (To appear.)

[24] Hoare, C.A.R. Recursive data structures. International Journal of Computer and Information Sciences, 1.2, (June 1975), 105-132.

[25] Kleene, S.C. Introduction to Metamathematics. D. Van Nostrand, Princeton, NJ, 1952.

[26] Kleene, S.C. Formalized recursive functionals and formalized realizability. Memoirs of the American Math. Society, No. 89, 1969.

[27] Kreisel, G. Generalized inductive definitions, section III. Standard report on the foundations of analysis (mimeographed), 1963.

[28] Knuth, D.E. The Art of Computer Programming, Vol. I. Addison-Wesley, Reading, 1968.

[29] MacQueen, D.B., Plotkin, Gordon D. and Sethi, R. An Ideal Model for Recursive Polymorphic Types. 11th ACM Symp. on Principles of Programming Languages, (1984) 165-174.

[30] MacQueen, D.B., and Sethi, R. A semantic model of types for applicative languages. ACM Symp. on LISP and Functional Programming, 1982, 243-252.

[31] Martin-Löf, Per. Constructive mathematics and computer programming. In 6th International Congress for Logic, Methodology and Philosophy of Science, North-Holland, Amsterdam, 1982.

[32] Martin-Löf, P. Hauptsatz for the intuitionistic theory of iterated inductive definitions. In Proceedings of the Second Scandinavian Logic Symposium, J.E. Fenstad (Ed.), North-Holland, Amsterdam, 1971, 179-216.

[33] McCarty, David C. Realizability and recursive mathematics. Computer Science Dept. Tech. Rpt. CMU-CS-84-131, Carnegie-Mellon University (1984).

[34] McGettrick, A.D. Algol 68, A First and Second Course. Cambridge University Press, Cambridge, 1978.

[35] Meyer, Albert R. What is a model of the lambda calculus? Information and Control, 52, (1982), 87-122.

[36] Moschovakis, Yiannis N. Elementary Induction on Abstract Structures. North Holland, London, 1974.

[37] Plotkin, Gordon D. T $T^{w}$ as a universal domain. J. Computer and System Sciences, 17, (1978), 209-236.

[38] Plotkin, Gordon D. Private communication.

[39] PRL Staff, The. Constructing proofs: An introduction to the Nupr! proof development system. Computer Science Department, Cornell University, January 1985.

[40] Quine, Willard Van Orman. Set Theory and Its Logic. Harvard University Press, Cambridge, 1963.

[41] Reynolds, John C. Types, abstraction, and parametric polymorphism. Information Processing 89, IFIP, North Holland Publishers, 1983, 513-523.

[42] Russell, B. Mathematical logic as based on a theory of types. Am. J. of Math., 90, (1908), 222-262.

[43] Scott, Dana. Data types as lattices. SIAM Journal on Computing, 5:3, (September 1976) 522587. 
[44] Scott, Dana. The lambda calculus, some models, some philosophy. The Kleene Symposium [eds., J. Barwise, et al.], North-Holland, 1980, 381-421.

[45] Scott, Dana. Constructive validity. In Symposium on Automatic Demonstration, Lecture Notes in Mathematics 125, Springer-Verlag, 1970, 237-275.

[46] Stenlund, S. Combinators, Lambda-terms, and Proof-Theory. D. Reidel, Dordrecht, 1972, 183.

[47] Stoy, Joseph E. Denotational Semantics: The Scott-Strachey Approach to Programming Language Theory. MIT Press, Cambridge, MA, 1977.

[48] Tait, William W. Intensional interpretation of functionals of finite type. J. Symbolic Logic, 32:2, (June 1967), 198-212.

[49] van Heijenoort, Jean. From Frege to Gödel: A source book in mathematical logic, 1879-1931, Harvard University Press, Cambridge (1967).

[50] van Wijngaarden, A.B.J. et al. Revised report on the algorithmic language ALGO 68. Acta Informatica, 5, (1975), 1-236. (Also a Supplement to ALGO BULLETIN, University of Alberta, 1974.) 